\title{
Glyoxysomal Nature of Microbodies Complexed with Lipid Globules in Botryosphaeria dothidea
}

\author{
Ki Woo Kim, Eun Woo Park, and Kyung Soo Kim
}

\begin{abstract}
First author: National Instrumentation Center for Environmental Management, Seoul National University, Seoul 151-742, Korea; second author: Department of Agricultural Biotechnology, Seoul National University, Seoul 151-742, Korea; and third author: Department of Plant Pathology, University of Arkansas, Fayetteville 72701.
\end{abstract} Accepted for publication 30 April 2004.

\begin{abstract}
Kim, K. W., Park, E. W., and Kim, K. S. 2004. Glyoxysomal nature of microbodies complexed with lipid globules in Botryosphaeria dothidea. Phytopathology 94:970-977.

The glyoxysomal nature of microbodies was determined in Botryos-
phaeria dothidea hyphae based on morphology and in situ enzyme
characteristics by transmission electron microscopy and cytochemistry.
Bound by a single membrane, microbodies had a homogeneous matrix
and varied in size ranging from 200 to $400 \mathrm{~nm}$ in diameter. Microbodies
often had crystalline inclusions that consisted of parallel arrays of fine
tubules in their matrices. Microbodies and lipid globules were placed
in close association with each other, forming microbody-lipid globule
complexes in hyphae. The cytochemical activities of catalase and malate
\end{abstract}

ABSTRACT

Microbodies are morphologically defined organelles that are bound by a single membrane and have a homogeneous granular matrix in eukaryotic cells $(16,20)$. They often contain inclusions in the matrix and are topographically associated with other organelles. Based on their functions, microbodies in higher plants are usually classified into two groups: (i) peroxisomes for photorespiration in photosynthetic leaves and (ii) glyoxysomes for lipid utilization in germinating seeds (20). All groups of microbodies contain catalase (EC 1.11.1.6) which detoxifies hydrogen peroxide to oxygen and water. The glyoxysomes are a site for the glyoxylate cycle, which leads to the synthesis of succinate from acetyl-CoA derived from fatty acids, and the key enzymes are (i) isocitrate lyase (EC 4.1.3.1) which splits isocitrate to succinate and glyoxylate, and (ii) malate synthase (EC 4.1.3.2) which converts glyoxylate and acetyl-CoA to malate.

In fungi, microbodies have been intensively studied as to the basis of their occurrence and peroxisomal biochemistry (16); however, little information is available on the glyoxysomal nature of microbodies in plant-pathogenic fungi. However, there is growing evidence that glyoxysomes and the glyoxylate cycle are involved in critical processes including appressorium formation of plant-pathogenic fungi $(12,27)$. The activity and presence of catalase in plant-pathogenic fungi are highly relevant to pathogenicity and infection processes, because plant disease resistance may include an increase in hydrogen peroxide concentration within host cells (14). The role of a fungal extracellular catalase in pathogenicity and suppression of host defense responses in Claviceps purpurea-rye interaction has been reported (4).

Corresponding author: E. W. Park; E-mail address: ewpark@snu.ac.kr

Publication no. P-2004-0714-02R

(C) 2004 The American Phytopathological Society synthase were localized in microbodies, showing intense electron density of the organelle. In addition, immunogold labeling detected the presence of catalase in a multivesicular body-like organelle and the cell wall as well as in the matrix and crystalline inclusion of microbodies, supporting the enzyme secretion outward. Meanwhile, isocitrate lyase was localized only in matrices of microbodies. These results suggest that the microbodies complexed with lipid globules in $B$. dothidea hyphae are functionally defined as glyoxysomes which may enable the fungus to survive latent periods using lipids via the glyoxylate cycle and catalase secretion.

Additional keywords: active oxygen species, Fusicoccum aesculi, latent infection, peroxisomes, white rot.
The ascomycetous fungus Botryosphaeria dothidea Ces. \& De Not. (anamorph Fusicoccum aesculi Corda) causes white rot, one of the most destructive diseases of apple (Malus domestica Borkh.) in humid areas (25). The disease produces a soft and light-colored rot on fruits and extensive cankers on limbs, eventually leading to tree death. The fungus forms appressoria on the fruit surface or enters fruits through natural openings such as lenticels and surface cracks (10). Apple fruits can be infected by conidia within 7 weeks after petal fall, and symptoms usually become noticeable 6 to 8 weeks before harvest (21). It is generally accepted that fungitoxic compounds including benzoic acid in immature fruits and fruit substrates unfavorable to the fungal growth are responsible for suppressing pathogen invasion and delaying symptom appearance (24). Our previous study (11) revealed the frequent occurrence of microbody-lipid globule complexes in B. dothidea hyphae invading immature apple fruits. Based on the presence of crystalline inclusions in the matrix and topographical association with lipid globules, the microbodies were considered to be glyoxysomes with catalase crystals. However, the biochemical nature of these organelles was not cytochemically characterized. Specific cytochemical localization of enzymes unique to glyoxysomes can be used for in situ identification of glyoxysomes. Either isocitrate lyase or malate synthase might be suitable for this purpose, because they are the key enzymes of the glyoxylate cycle occurring in glyoxysomes. In situ activity and subcellular localization of glyoxysomal enzymes through enzyme cytochemistry and immunocytochemistry will provide a better understanding of the microbody-lipid globule complexes and the fungal survival mechanisms underlying dormant hyphal growth and latent infections in immature apple fruits. The objectives of this study were to (i) investigate the morphology and in situ enzyme characteristics of the microbodies and (ii) provide evidence for the glyoxysomal nature of microbodies in $B$. dothidea hyphae. 


\section{MATERIALS AND METHODS}

Fungal isolate and culture conditions. An isolate (BD-3) of $B$. dothidea was obtained from a naturally infected apple fruit showing typical white rot symptoms. The isolate was grown and stored on autoclaved barley (Hordeum vulgare L.) grains at $25^{\circ} \mathrm{C}$ (9). The isolate was transferred to potato dextrose agar (PDA) plates and grown under a 12-h photoperiod of fluorescent light at $25^{\circ} \mathrm{C}$. The cultures were subjected to specimen preparation for transmission electron microscopy.

Hyphal ultrastructure. Three types of $B$. dothidea hyphae were collected as follows: (i) actively growing tips from the margin of a 3-day-old culture grown on PDA, (ii) nonmarginal hyphae from a 30-day-old culture grown on PDA, and (iii) nonmarginal hyphae from a 30-day-old culture grown on autoclaved barley grains. The hyphal tips and samples taken from either of the 30-day-old cultures were designated in this study as young and old hyphae, respectively. Squares of young hyphae (each $2 \times$ $2 \mathrm{~mm}^{2}$ with approximately $1 \mathrm{~mm}$ thickness of underlying agar blocks) were excised with a sterile razor blade from the margin of actively growing 3-day-old PDA cultures. Squares of the same size were obtained from the center of 30-day-old PDA plates and autoclaved barley grains. The specimens were fixed with modified Karnovsky's fixative (8) consisting of $2 \%$ (vol/vol) glutaraldehyde and $2 \%$ (vol/vol) paraformaldehyde in $50 \mathrm{mM}$ sodium cacodylate buffer $\left(\mathrm{pH} \mathrm{7.2)}\right.$ ) at $4^{\circ} \mathrm{C}$ overnight and washed with the same buffer three times each for $10 \mathrm{~min}$. The specimens were postfixed with $1 \%(\mathrm{wt} / \mathrm{vol})$ osmium tetroxide in the same buffer at $4^{\circ} \mathrm{C}$ for $2 \mathrm{~h}$ and washed briefly with distilled water two times. The postfixed specimens were en bloc stained with $0.5 \%$ (wt/vol) uranyl acetate at $4^{\circ} \mathrm{C}$ overnight and dehydrated in a graded ethanol series: $30,50,70,80,95$, and $100 \%$, and three times in $100 \%$ each for $10 \mathrm{~min}$. The specimens were further treated with propylene oxide two times each for $30 \mathrm{~min}$ as a transitional fluid and embedded in Spurr's resin. Ultrathin sections (approximately $50 \mathrm{~nm}$ thick) were cut with a diamond knife using an ultramicrotome (MT-X; RMC Inc., Tucson, AZ). The ultrathin sections were mounted on copper grids and stained with $2 \%$ uranyl acetate and Reynolds' lead citrate (23) each for $7 \mathrm{~min}$. They were examined with a transmission electron microscope (JEM-1010; JEOL Ltd., Tokyo) operated at an accelerating voltage of $80 \mathrm{kV}$. More than 10 ultrathin sections per specimen, from a total of three specimens per hyphal type, were examined with the microscope.

Enzyme cytochemistry. Cytochemical localization of catalase activity in hyphae was carried out by the 3,3'-diaminobenzidine tetrahydrochloride (DAB) staining method $(4,31)$ with slight modifications as follows. Squares of the 30-day-old hyphae grown on PDA were sampled as described previously. The specimens were fixed with modified Karnovsky's fixative consisting of $0.5 \%$ ( $\mathrm{vol} / \mathrm{vol}$ ) glutaraldehyde and $4 \%$ (vol/vol) paraformaldehyde in $0.05 \mathrm{M}$ sodium cacodylate buffer $\left(\mathrm{pH} \mathrm{7.2)}\right.$ ) at $4^{\circ} \mathrm{C}$ for $2 \mathrm{~h}$ and washed as described previously. They were then incubated in $0.05 \mathrm{M}$ sodium cacodylate buffer $(\mathrm{pH}$ 9.5) containing $0.2 \%$ (wt/vol) DAB and $0.06 \%$ (vol/vol) hydrogen peroxide at $37^{\circ} \mathrm{C}$ for $30 \mathrm{~min}$. This incubation step was performed with gentle shaking under continuous darkness conditions and repeated two times. Other specimens were also incubated in the incubation medium lacking hydrogen peroxide as a negative control. The specimens were then washed in $0.05 \mathrm{M}$ sodium cacodylate buffer $(\mathrm{pH} 7.2)$ three times each for $10 \mathrm{~min}$. The specimens were postfixed, further processed, embedded in LR white resin, and examined as described previously.

To localize malate synthase activity in hyphae, another cytochemical staining method (30) was employed with slight modifications as follows. Squares of the 30-day-old hyphae grown on PDA were fixed and washed as described in the catalase cytochemistry study. They were then preincubated in $0.01 \mathrm{M}$ potassium ferricyanide and $0.02 \mathrm{M}$ sodium cacodylate buffer $(\mathrm{pH} 7.2)$ at room temperature $\left(20\right.$ to $\left.24^{\circ} \mathrm{C}\right)$ under continuous darkness conditions. After $30 \mathrm{~min}$, they were immersed in an incubation medium (30) with $0.065 \mathrm{M}$ sodium cacodylate buffer ( $\mathrm{pH} 7.2)$. The incubation step was performed at $37^{\circ} \mathrm{C}$ under continuous darkness conditions for $30 \mathrm{~min}$ and repeated once. Other specimens were also treated with the same incubation medium, except lacking acetyl-CoA, as a negative control. The specimens were postfixed and further processed as described in the catalase cytochemistry study.

Western blot analysis. The fungus was grown in potato dextrose broth at $25^{\circ} \mathrm{C}$ for 21 days. Whole protein extracts were prepared by homogenizing mycelia with liquid nitrogen in a buffer containing $50 \mathrm{mM}$ Tris- $\mathrm{HCl}$ (pH 7.4), $1 \mathrm{mM}$ EGTA, $0.2 \%$ Triton $\mathrm{X}-100,1 \mathrm{mM}$ phenylmethyl sulfonyl fluoride, and $10 \mu \mathrm{g} / \mathrm{ml}$ each of leupeptin and pepstatin (7). The homogenates were clarified by centrifugation at $10,000 \times g$ for $10 \mathrm{~min}$ at $4^{\circ} \mathrm{C}$ and then at $25,000 \times$ $g$ for $60 \mathrm{~min}$ at $4^{\circ} \mathrm{C}$. The proteins in supernatants were separated by sodium dodecyl sulfate-polyacrylamide gel electrophoresis (SDS-PAGE) (12\% acrylamide gel) and transferred to a polyvinylidene difluoride membrane. The membrane was washed with $0.1 \mathrm{M}$ Tris- $\mathrm{HCl}(\mathrm{pH} \mathrm{8.0)}$ and treated with a blocking solution containing $5 \%(\mathrm{wt} / \mathrm{vol})$ nonfat dry milk in Tris-buffered saline (TBST; $100 \mathrm{mM}$ Tris- $\mathrm{HCl}$ and $0.05 \%$ Tween 20, $\mathrm{pH} 8.0$ ) at room temperature for $1.5 \mathrm{~h}$. The membrane was then incubated for $1.5 \mathrm{~h}$ with rabbit polyclonal antisera of (i) catalase (Polysciences Inc., Warrington, PA) raised against bovine liver catalase and (ii) isocitrate lyase raised against spinach isocitrate lyase diluted $1: 1,000$ with the $0.2 \%$ blocking solution. After briefly washing two times with TBST, the membrane was further treated for $1.5 \mathrm{~h}$ with alkaline phosphatase-conjugated goat anti-rabbit immunoglobulin G (IgG) (Pierce Biotechnology Inc., Rockford, IL) diluted $1: 1,000$ with the $0.2 \%$ blocking solution. The membrane was washed with TBST and stained with a combination of nitroblue tetrazolium chloride and 5-bromo-4-chloro-3'-indolyphosphate $p$-toluidine salt to visualize antigen-antibody complexes on the blotted membrane.

Immunocytochemistry. To localize catalase and isocitrate lyase in hyphae, immunogold labeling was performed using polyclonal antisera raised against each enzyme. Squares of old hyphae grown on PDA were fixed and washed as described in the enzyme cytochemistry study. After en bloc staining with $0.5 \%$ uranyl acetate at $4^{\circ} \mathrm{C}$ overnight, they were dehydrated in a graded ethanol series $(30,50,70,80$, and $95 \%)$ each for 10 min and embedded in LR white resin. The reaction conditions and optimum dilution ratios of immunogold labeling mixtures were determined in preliminary studies. Ultrathin sections mounted on Formvar-coated nickel grids were treated with a blocking solution containing 5\% (wt/vol) nonfat dry milk in Tris-buffered saline (TBS; $50 \mathrm{mM}$ Tris- $\mathrm{HCl}$ and $150 \mathrm{mM} \mathrm{NaCl}, \mathrm{pH} \mathrm{7.2)} \mathrm{at} \mathrm{room} \mathrm{temperature} \mathrm{for}$ $10 \mathrm{~min}$. The grids were incubated in a moist chamber overnight with the rabbit polyclonal antisera of catalase and isocitrate lyase diluted 1:250 with the blocking solution. After briefly washing three times with TBS, they were further treated for $1 \mathrm{~h}$ with goat anti-rabbit IgG conjugated with $10 \mathrm{~nm}$ colloidal gold particles (Sigma Chemical, St. Louis) diluted 1:50 with TBS. They were then washed thoroughly with TBS followed by sterilized distilled water, stained, and examined as described previously. Negative control experiments were also performed to prove the specificity of the labeling through the replacement of the primary antisera with preimmune serum of rabbit.

\section{RESULTS}

Hyphal ultrastructure. Transmission electron microscopy of young hyphae revealed numerous ribosomes in the cytoplasm enclosed by an electron-translucent cell wall (Fig. 1A). The hyphae contained nuclei, mitochondria, and vacuoles in cytoplasm. Microbodies were usually round in cross sections, bound by a single 
membrane, and had a homogeneous matrix in young hyphae. Unlike mitochondria with cristae projecting into matrices, microbodies did not have internal membrane systems in such matrices. Old hyphae grown on PDA had thicker cell walls than young hyphae, and the overall appearance of the cell wall from old hyphae differed from that of the cell wall from young hyphae (Fig. 1B). The cytoplasm had large vacuoles, lipid globules, and mitochondria. Microbodies varied in size, ranging from 200 to $400 \mathrm{~nm}$ in diameter, and often had crystalline inclusions which consisted of parallel arrays of fine tubules in their matrices (Fig. 1C). In addition, microbodies and lipid globules were placed in close association with each other, forming microbody-lipid globule complexes in old hyphae grown on either PDA plates or autoclaved barley grains (Fig. 1D). The morphology and size of microbodies in old hyphae grown on autoclaved barley grains were similar to those of the organelle found in young or old hyphae grown on PDA.

Enzyme cytochemistry. Microbody-lipid globule complexes were found in old hyphae grown on PDA (Fig. 2A). However, it was common to observe more electron-dense microbodies after the DAB staining with the complete incubation medium including hydrogen peroxide than when staining in medium lacking hydrogen peroxide (compare Fig. 2B with 2A). The staining of catalase activity was uniform throughout the matrix of microbodies, so even crystalline inclusions were nearly indiscernible within some intensely stained microbodies (Fig. 2C). In addition to microbodies, some mitochondria exhibited cytochemical staining (Fig. 2D).
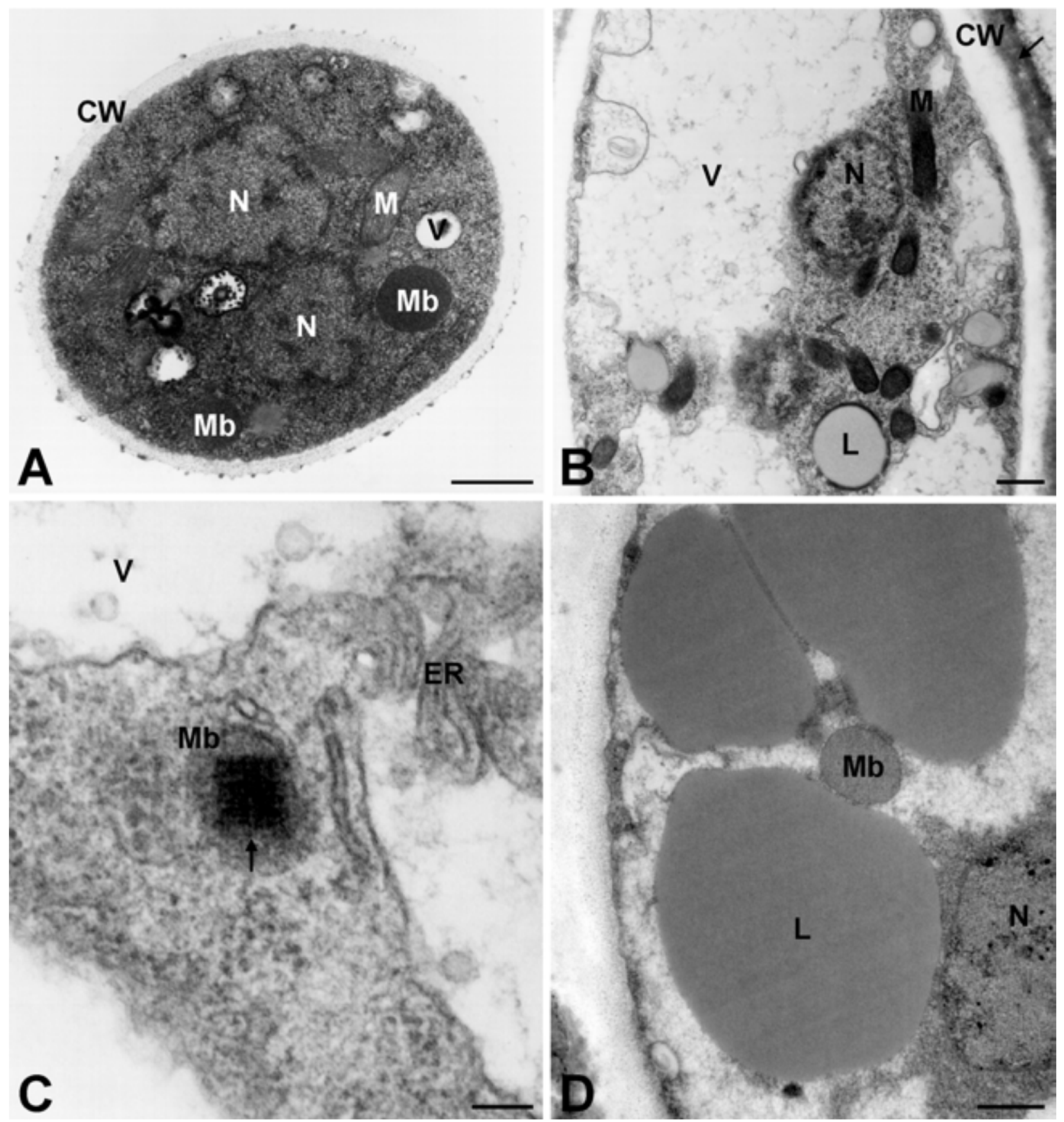

Fig. 1. Ultrastructure of Botryosphaeria dothidea hyphae. A, A section near a hyphal tip grown on potato dextrose agar (PDA). The cytoplasm was filled with numerous ribosomes. $\mathrm{CW}=$ cell wall, $\mathrm{M}=$ mitochondrion, $\mathrm{Mb}=$ microbody, $\mathrm{N}=$ nucleus, $\mathrm{V}=$ vacuole. $\mathrm{Bar}=500 \mathrm{~nm}$. B, A 30-day-old hypha grown on PDA. The cytoplasm was mainly characterized by large vacuoles $(\mathrm{V})$ and lipid globules $(\mathrm{L})$. Arrow indicates electron-dense outer layer of the cell wall. Bar $=500 \mathrm{~nm}$. C, A microbody in the 30-day-old hypha. The organelle contained a crystalline inclusion (arrow) in the matrix. ER = endoplasmic reticulum. Bar $=100 \mathrm{~nm}$. D, A 30-day-old hypha grown on autoclaved barley grains. Microbody-lipid globule complexes were often found in cytoplasm. Bar $=300 \mathrm{~nm}$. 
Hyphae had microbodies and mitochondria with normal electron density when the incubation medium for malate synthase cytochemistry lacked acetyl-CoA (Fig. 3A). However, incubations with the complete incubation medium including acetyl-CoA for malate synthase cytochemistry resulted in the intense and uniform deposition of electron-dense reaction products throughout the matrix of the microbodies and mitochondria with cristae (Fig. 3B).

Western blot analysis. The SDS-PAGE of whole protein extracts showed that a number of soluble proteins were induced and accumulated in the mycelium. The anti-catalase antiserum raised against bovine liver catalase detected two major protein bands in western blots (Fig. 4A). The molecular masses of these immuno- reactive proteins were estimated to be approximately 40 and $45 \mathrm{kDa}$. In addition, two major protein bands at molecular masses of approximately 50 and $60 \mathrm{kDa}$ were detected with the antiisocitrate lyase antiserum raised against spinach isocitrate lyase (Fig. 4B).

Immunocytochemistry. As usual in the nonosmicated specimens embedded in LR white resin, ultrathin sections showed membrane systems of low contrast. The control experiment gave negative results, with only nonspecifically scattered gold particles on ultrathin sections (Fig. 5A). When ultrathin sections were incubated with the anti-catalase antiserum, gold particles were specifically localized in both crystalline inclusions and matrices
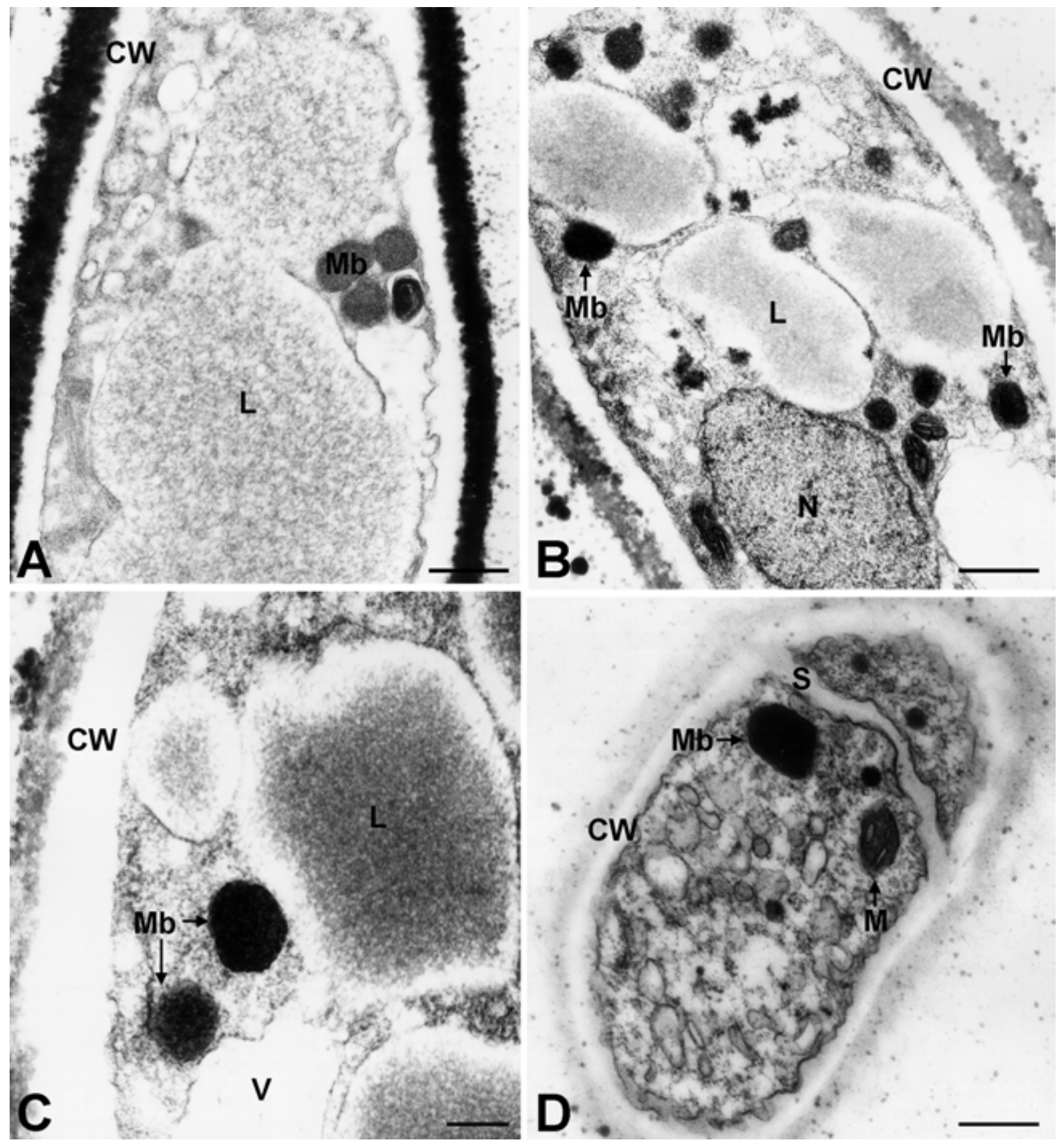

Fig. 2. In situ localization of catalase activity in Botryosphaeria dothidea hyphae. A, A 30-day-old hypha after the 3,3'-diaminobenzidine tetrahydrochloride (DAB) staining with the incubation medium lacking hydrogen peroxide (negative control). Bar $=500 \mathrm{~nm}$. B, A 30-day-old hypha after the DAB staining with the complete incubation medium including hydrogen peroxide. Microbodies were more electron-dense than those in A. Bar $=400 \mathrm{~nm}$. C, Higher magnification of microbodies in a 30-day-old hypha after the DAB staining with the complete incubation medium. Even crystalline inclusions were indiscernible within intensely stained microbodies. Bar $=200 \mathrm{~nm}$. D, A 30-day-old hypha after the DAB staining with the complete incubation medium. Some mitochondria showed cytochemical staining. $\mathrm{S}=$ septum. $\mathrm{Bar}=500 \mathrm{~nm}$. 
of microbodies (Fig. 5B). The gold labeling was also evident in a multivesicular body-like organelle containing small bubble-like vesicles and in the cell wall. The density of labeling indicated about $13 \%$ of the gold particles localized to microbodies in the micrograph (Fig. 5B).

When ultrathin sections were incubated with the anti-isocitrate lyase antiserum, gold particles were specifically localized only in the matrices of microbodies (Fig. 5C). Crystalline inclusions were not labeled with gold particles, even though the inclusions were present in the gold particle-labeled matrices of microbodies. In some cases, the matrices of microbodies were not labeled with gold particles when crystalline inclusions occupied a majority of the matrix of the organelle in ultrathin sections. About $62 \%$ of gold particles were localized to microbodies in the micrograph (Fig. 5C). Two different quadrangular crystalline inclusions were often found in the matrices of microbodies, where only the matrices were specifically labeled with gold particles (Fig. 5D). Occasional nonspecific gold particles were observed in other cell components in addition to microbodies.

\section{DISCUSSION}

Plant-pathogenic fungi are exposed to limited nutrient conditions during the saprophytic or parasitic phase, and thus need to use storage compounds for survival. They commonly use lipids, the dominant form of stored carbon, and coordinate the topographical associations of organelles for the efficient production of energy $(11,27)$. This study demonstrated the glyoxysomal nature of microbodies in B. dothidea hyphae by the criteria of the exami-

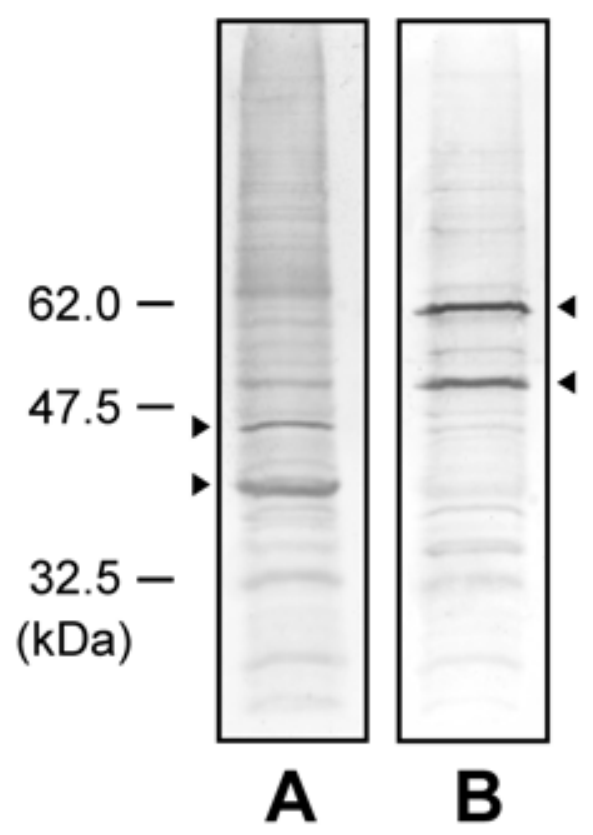

Fig. 4. Western blot analysis of catalase and isocitrate lyase in Botryosphaeria dothidea hyphae. A, Detection of catalase from 21-day-old hyphae. Note the immunoreactive protein bands at the molecular masses of approximately 40 and $45 \mathrm{kDa}$. B, Detection of isocitrate lyase from 21-day-old hyphae. Note the immunoreactive protein bands at the molecular masses of approximately 50 and $60 \mathrm{kDa}$. The approximate sizes of the proteins are shown at the left. Arrowheads indicate the immunoreactive protein bands in each lane.

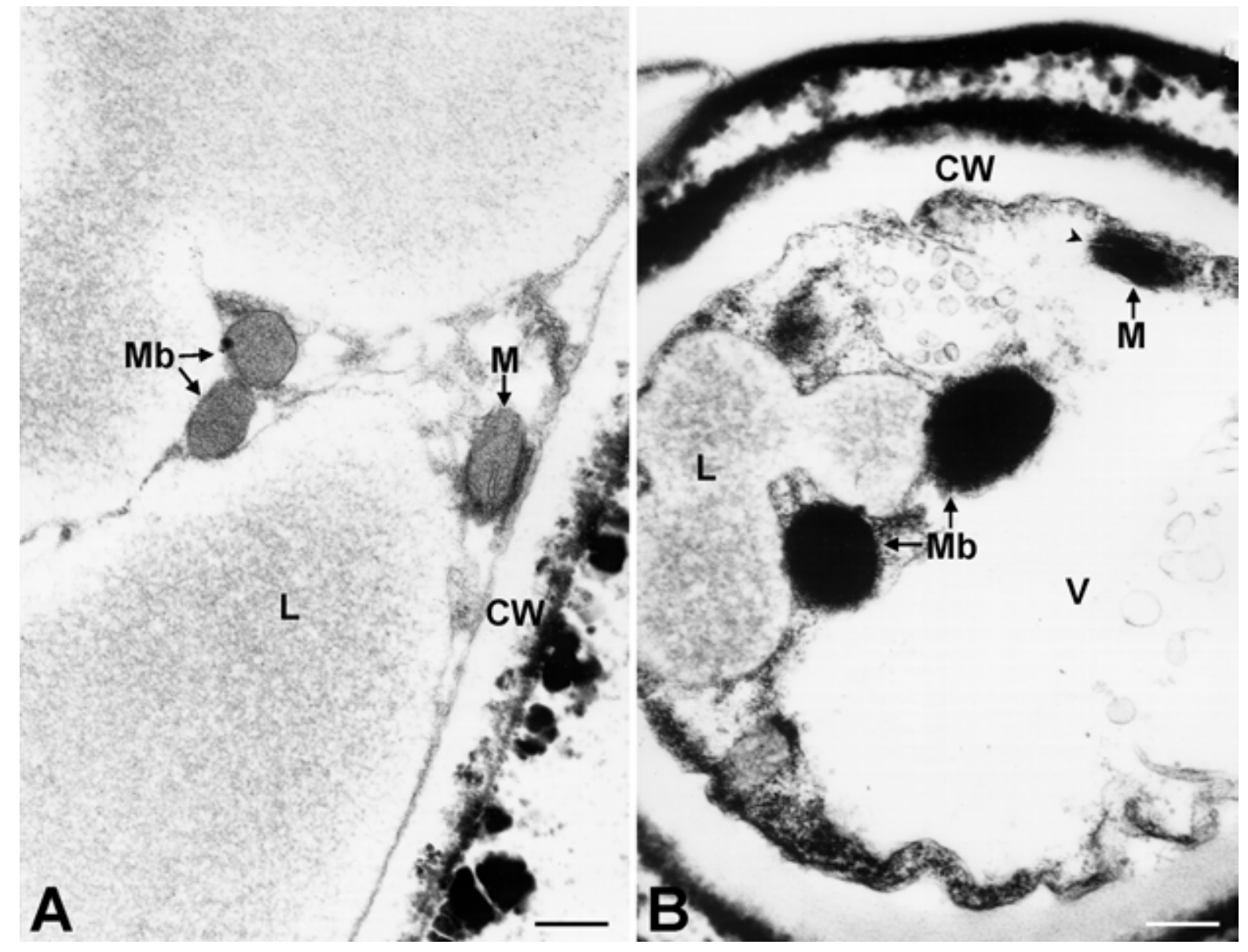

Fig. 3. In situ localization of malate synthase activity in Botryosphaeria dothidea hyphae. A, A 30-day-old hypha after the cytochemical staining with the incubation medium lacking acetyl-CoA (negative control). Microbodies ( $\mathrm{Mb}$ ) and mitochondria $(\mathrm{M})$ showed normal electron density. B, A 30-day-old hypha after the cytochemical staining with the complete incubation medium including acetyl-CoA. Microbodies and a mitochondrion with cristae (arrowhead) were more electron-dense than those in A. Bars $=200 \mathrm{~nm}$. 
nation of organellar morphology and cytochemistry of enzymes unique to glyoxysomes. The morphological characteristics such as shape, size, and matrix feature of the microbodies were consistent with those of the glyoxysomes in other fungi (16). In particular, the proximity of microbodies with lipid globules in hyphae was strikingly similar to that found in zoospores of chytrids or germinating seeds, where microbodies (glyoxysomes) were involved in lipid utilization via the glyoxylate cycle $(20$, 22,28). Furthermore, enzyme cytochemistry and immunocytochemistry provided evidence for in situ activity and subcellular localization of glyoxysomal enzymes in microbodies of hyphae, respectively. These results suggest that the microbodies com- plexed with lipid globules in $B$. dothidea hyphae are functionally defined as glyoxysomes, where glyoxysomal enzymes are biochemically active for the glyoxylate cycle to be a metabolic pathway in gluconeogenesis. To our knowledge, this is the first report of the in situ activity and subcellular localization of glyoxysomal enzymes in plant-pathogenic fungi.

Microbodies were invariably found in cytoplasm regardless of the hyphal types tested; however, crystalline inclusions in the organellar matrix were often observed in old hyphae. As inferred from the previous observation of the inclusions in $B$. dothidea hyphae invading immature apple fruits (11), it is likely that the inclusion formation would increase while nutrients in culture or
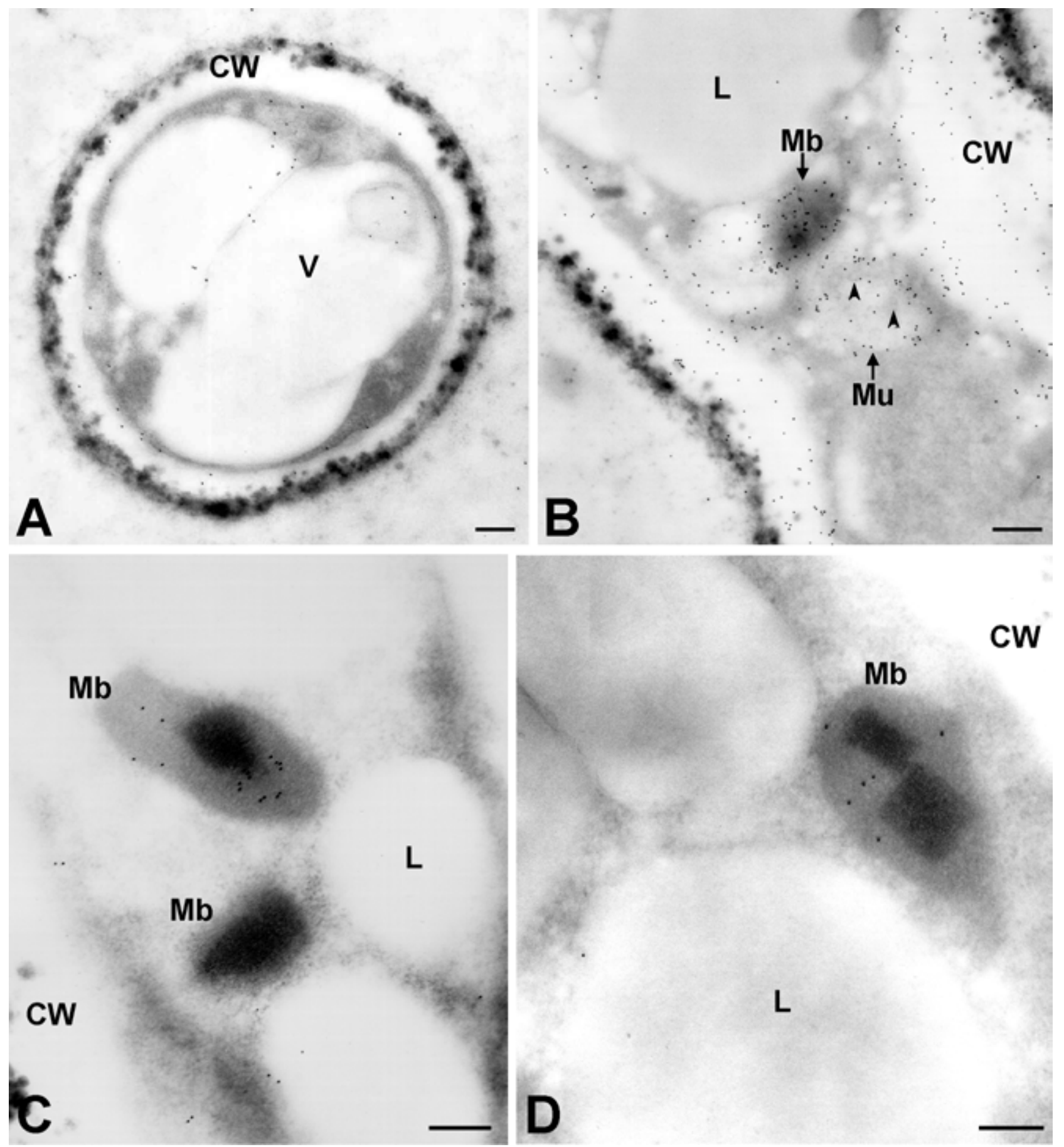

Fig. 5. Subcellular localization of catalase and isocitrate lyase in Botryosphaeria dothidea hyphae. A, A 30-day-old hypha treated with the preimmune serum of rabbit (negative control). Gold particles were nonspecifically labeled on the ultrathin section. B, Immunogold labeling of catalase in a 30-day-old hypha. Note the gold particles labeled in a microbody (Mb), a multivesicular body-like organelle (Mu), and a cell wall (CW). Arrowheads indicate bubble-like vesicles. C and $\mathbf{D}$, Immunogold labeling of isocitrate lyase in a 30-day-old hypha. Gold particles were specifically labeled in the matrix of a microbody $(\mathrm{Mb})$. Bars $=200 \mathrm{~nm}$. 
plant substrate were being depleted during hyphal growth. Also, such limited nutrient conditions might favor the formation of microbody-lipid globule complexes, considering the frequent occurrence of the complexes in old hyphae. The cytochemical localization of catalase activity indicated that the enzyme was biochemically active in microbodies, as reported from other fungi $(4,31)$. In the presence of exogenously added hydrogen peroxide, catalase has been known to mediate the oxidation of DAB into an osmiophilic polymer, which reduces osmium tetroxide, resulting in electron-dense deposits (6). The activity of malate synthase was also localized in microbodies, suggesting that the enzyme would be biochemically active for the glyoxylate cycle to provide the fungus with energy and 4-carbon intermediates of the tricarboxylic acid cycle $(17,20)$. Considering the components of the incubation medium, the pronounced electron-dense materials in the malate synthase cytochemistry study would likely correspond to copper ferrocyanide (30). Meanwhile, the electron-dense staining and reactivity of mitochondria may be due to the peroxidative reaction of cytochrome c peroxidase or several mitochondrial heme proteins (29).

The immunocytochemistry detected the presence of catalase in matrices and crystalline inclusions of microbodies, where catalase activity was demonstrated by the DAB staining method. These findings support that the crystalline inclusions are composed of biochemically active catalase molecules that undergo crystallization in the matrix (26). Moreover, the immunogold labeling indicated the presence of catalase in a multivesicular body-like organelle and a cell wall, suggesting the enzyme secretion outward. This result was consistent with the observation that Claviceps purpurea catalase was secreted via fungal secretory pathway through multivesicular bodies (4). The distribution of gold particles on the nonmicrobody regions could be due to the extracellular secretion of catalase. Meanwhile, the specific location of isocitrate lyase in matrices of microbodies in this study implies that the glyoxylate cycle occurs in the microbodies, as also suggested from the in situ activity of malate synthase. The relatively high density of labeling also supported the specific localization of the enzyme in microbodies.

In addition, the specific cross-reactivity of heterologous antisera produced against catalase and isocitrate lyase with the corresponding enzymes of $B$. dothidea was shown in this study. Considering the specific labeling of gold particles, it is likely that catalase and isocitrate lyase of the fungus share a considerable number of epitopes with those of the heterologous hosts. Moreover, the results from the western blot analysis of catalase and isocitrate lyase supported that the antisera raised against the two enzymes from heterologous hosts had cross-reactivity with the fungal-derived proteins. In particular, the molecular mass of a protein band having the binding specificity with the anti-isocitrate lyase antiserum was estimated to be approximately $60 \mathrm{kDa}$, which was similar to that of isocitrate lyase from Aspergillus nidulans (3) and Magnaporthe grisea (32). The putative molecular masses of protein bands that strongly reacted with the anticatalase antiserum in this study were not in exact agreement with those of catalase from other fungi $(2,4,5)$. The discrepancy between the results can be explained by assuming that fungi contain multiple catalase genes that form a quite diverse group of enzymes with respect to their structures, locations, and functions (5). It is considered that the anti-catalase antiserum was mainly bound to the microbody-localized and extracellularly secreted catalase(s) from the entire catalase populations in $B$. dothidea. Future work remains to be done to characterize the biochemical nature of the glyoxysomal enzymes from $B$. dothidea that may have different isoforms or subunits in structure and conformation.

The putative involvement of glyoxysomes in the survival and pathogenicity of B. dothidea could be proposed in this study. One of the earliest and most effective responses of host plants after pathogen recognition is the oxidative burst, cascade generation of active oxygen species including superoxide radical, hydrogen peroxide, and hydroxyl radical (14). Hydrogen peroxide is closely associated with a variety of host defense responses such as cell wall fortification, hypersensitive response, and direct antimicrobial action $(1,14,19)$. To establish compatible interactions with host plants, $B$. dothidea might counteract the oxidative burst by secreting catalase as an enzymatic defense against hydrogen peroxide. Diffusible catalase could counter hydrogen peroxide acting on host cell walls at the host-parasite interface, suppressing hydrogen peroxide-mediated host defense responses. We suggest, therefore, that the glyoxysomes enable the fungus to endure latent periods and adverse conditions, such as nutrient deficiency and host defense responses, allowing the use of lipids via the glyoxylate cycle and catalase secretion until the fungus meets suitable nutrients. Although suppressed in immature fruits, $B$. dothidea is thought to survive unfavorable conditions and resume its active growth as the sugar content in fruits increases (13). The crucial roles of catalase and the glyoxylate cycle have been well established in the pathogenesis of human and plant diseases caused by fungi and bacteria, indicating the widespread utility of the enzyme and the pathway in microbial pathogenesis $(15,18,32,33)$.

In conclusion, this study increased our knowledge of the cytology and in situ enzyme characteristics of glyoxysomes in $B$. dothidea hyphae. The implication of glyoxysomes with fungal survival suggests that the organellar enzymes and glyoxylate cycle would perhaps present site-specific novel targets for the control or intervention of plant diseases caused by fungi. It remains to be elucidated in further studies whether the glyoxylate cycle is essential to the latent infection or endophytic colonization of host tissues by $B$. dothidea. Also, the knowledge of the developmental transition and regulation of enzyme activities and the occurrence of individual enzyme isoforms will enhance our understanding of the nature of fungal pathogenicity and survival.

\section{ACKNOWLEDGMENTS}

This study was supported by the Korea Science and Engineering Foundation. We thank J. B. Murphy in the Department of Horticulture at the University of Arkansas for providing the antiserum of isocitrate lyase; E. M. Martin and S. C. Goeke for technical assistance in electron microscopy; and J.-K. Kim and I. Hwang for technical assistance and comments on western blot analysis.

\section{LITERATURE CITED}

1. Bradley, D. J., Kjellbom, P., and Lamb, C. J. 1992. Elicitor- and woundinduced oxidative cross-linking of a proline-rich plant cell wall protein: A novel, rapid defense response. Cell 70:21-30.

2. Bussink, H.-J., and Oliver, R. 2001. Identification of two highly divergent catalase genes in the fungal tomato pathogen, Cladosporium fulvum. Eur. J. Biochem. 268:15-24.

3. De Lucas, J. R., Amor, C., Díaz, M., Turner, G., and Laborda, F. 1997. Purification and properties of isocitrate lyase from Aspergillus nidulans, a model enzyme to study catabolite inactivation in filamentous fungi. Mycol. Res. 101:410-414.

4. Garre, V., Tenberge, K. B., and Eising, R. 1998. Secretion of a fungal extracellular catalase by Claviceps purpurea during infection of rye: Putative role in pathogenicity and suppression of host defense. Phytopathology 88:744-753.

5. Goodwin, P. H., Li, J., and Jin, S. 2001. A catalase gene of Colletotrichum gloeosporioides f. sp. malvae is highly expressed during the necrotrophic phase of infection of round-leaved mallow, Malva pusilla. FEMS Microbiol. Lett. 202:103-107.

6. Hirai, K. I. 1975. Diaminobenzidine in cytochemistry. Acta Histochem. Cytochem. 8:31-40.

7. Juvvadi, P. R., Kuroki, Y., Arioka, M., Nakajima, H., and Kitamoto, K. 2003. Functional analysis of the calcineurin-encoding gene cnaA from Aspergillus oryzae: Evidence for its putative role in stress adaptation. Arch. Microbiol. 179:416-422.

8. Karnovsky, M. J. 1965. A formaldehyde-glutaraldehyde fixative of high osmolality for use in electron microscopy. J. Cell Biol. 27:137A-138A

9. Kim, K. W., and Park, E. W. 1998. Production and exudation of Botryos- 
phaeria dothidea conidia using cucumber disks and cereal media. (in Korean) Korean J. Plant Pathol. 14:46-51.

10. Kim, K. W., Park, E. W., and Ahn, K.-K. 1999. Pre-penetration behavior of Botryosphaeria dothidea on apple fruits. Plant Pathol. J. 15:223-227.

11. Kim, K. W., Park, E. W., Kim, Y. H., Ahn, K.-K., Kim, P. G., and Kim, K. S. 2001. Latency- and defense-related ultrastructural characteristics of apple fruit tissues infected with Botryosphaeria dothidea. Phytopathology 91:165-172.

12. Kimura, A., Takano, Y., Furusawa, I., and Okuno, T. 2001. Peroxisomal metabolic function is required for appressorium-mediated plant infection by Colletotrichum lagenarium. Plant Cell 13:1945-1957.

13. Kohn, F. C., Jr., and Hendrix, F. F. 1983. Influence of sugar content and $\mathrm{pH}$ on development of white rot on apples. Plant Dis. 67:410-412.

14. Lamb, C., and Dixon, R. A. 1997. The oxidative burst in plant disease resistance. Annu. Rev. Plant Physiol. Plant Mol. Biol. 48:251-275.

15. Lorenz, M. C., and Fink, G. R. 2001. The glyoxylate cycle is required for fungal virulence. Nature 412:83-86.

16. Maxwell, D. P., Armentrout, V. N., and Graves, L. B., Jr. 1977. Microbodies in plant pathogenic fungi. Annu. Rev. Phytopathol. 15:119-134.

17. McCammon, M. T., Veenhuis, M., Trapp, S. B., and Goodman, J. M. 1990. Association of glyoxylate and beta-oxidation enzymes with peroxisomes of Saccharomyces cerevisiae. J. Bacteriol. 172:5816-5827.

18. McKinney, J. D., Zu Bentrup, K. H., Muñoz-Elías, E. J., Miczak, A., Chen, B., Chan, W.-T., Swenson, D., Sacchettini, J. C., Jacobs, W. R., Jr., and Russell, D. G. 2000. Persistence of Mycobacterium tuberculosis in macrophages and mice requires the glyoxylate shunt enzyme isocitrate lyase. Nature 406:735-738.

19. Mellersh, D. G., Foulds, I. V., Higgins, V. J., and Heath, M. C. 2002. $\mathrm{H}_{2} \mathrm{O}_{2}$ plays different roles in determining penetration failure in three diverse plant-fungal interactions. Plant J. 29:257-268.

20. Olsen, L. J., and Harada, J. J. 1995. Peroxisomes and their assembly in higher plants. Annu. Rev. Plant Physiol. Plant Mol. Biol. 46:123-146.

21. Parker, K. C., and Sutton, T. B. 1993. Susceptibility of apple fruit to Botryosphaeria dothidea and isolate variation. Plant Dis. 77:385-389.
22. Powell, M. J. 1976. Ultrastructure and isolation of glyoxysomes (microbodies) in zoospores of the fungus Entophlyctis sp. Protoplasma 89:1-27.

23. Reynolds, E. S. 1963. The use of lead citrate at high $\mathrm{pH}$ as an electronopaque stain in electron microscopy. J. Cell Biol. 17:208-212.

24. Seng, J. M., Saindrenan, P., and Bompeix, G. 1985. Induction of Nectria galligena mutants resistant to benzoic acid and study of their aggressiveness towards to immature apples. J. Gen. Microbiol. 131:1863-1866.

25. Sutton, T. B. 1990. White rot. Pages 16-18 in: Compendium of Apple and Pear Diseases. A. L. Jones and H. S. Aldwinckle, eds. The American Phytopathological Society, St. Paul, MN.

26. Tenberge, K. B., and Eising, R. 1995. Immunogold labelling indicates high catalase concentrations in amorphous and crystalline inclusions of sunflower (Helianthus annuиs L.) peroxisomes. Histochem. J. 27:184-195.

27. Thines, E., Weber, R. W. S., and Talbot, N. J. 2000. MAP kinase and protein kinase A-dependent mobilization of triacylglycerol and glycogen during appressorium turgor generation by Magnaporthe grisea. Plant Cell 12:1703-1718.

28. Titus, D. E., and Becker, W. M. 1985. Investigation of the glyoxysomeperoxisome transition in germinating cucumber cotyledons using doublelabel immunoelectron microscopy. J. Cell Biol. 101:1288-1299.

29. Todd, M. M., and Vigil, E. L. 1972. Cytochemical localization of peroxidase activity in Saccharomyces cerevisiae. J. Histochem. Cytochem. 20: 344-349.

30. Trelease, R. N., Becker, W. M., and Burke, J. J. 1974. Cytochemical localization of malate synthase in glyoxysomes. J. Cell Biol. 60:483-495.

31. Van Dijken, J. P., Veenhuis, M., Vermeulen, C. A., and Harder, W. 1975. Cytochemical localization of catalase activity in methanol-grown Hansenula polymorpha. Arch. Microbiol. 105:261-267.

32. Wang, Z.-Y., Thornton, C. R., Kershaw, M. J., Debao, L., and Talbot, N. J. 2003. The glyoxylate cycle is required for temporal regulation of virulence by the plant pathogenic fungus Magnaporthe grisea. Mol. Microbiol. 47:1601-1612.

33. Xu, X. Q., and Pan, S. Q. 2000. An Agrobacterium catalase is a virulence factor involved in tumorigenesis. Mol. Microbiol. 35:407-414. 